\title{
THE IMPLICIT LEARNING OF MAPPINGS BETWEEN FORMS AND CONTEXTUALLY DERIVED MEANINGS
}

\author{
Janny H. C. Leung \\ The University of Hong Kong
}

\author{
John N. Williams \\ University of Cambridge
}

\begin{abstract}
The traditional implicit learning literature has focused primarily on the abstraction of statistical regularities in form-form connections. More attention has been recently directed toward the implicit learning of form-meaning connections, which might be crucial in the acquisition of natural languages. The current article reports evidence for implicit learning of a mapping between a novel set of determiners and thematic roles, obtained using a newly developed reaction time methodology. The results conclude that contextually derived form-meaning connections might be implicitly learned.
\end{abstract}

The phenomenon of implicit learning-that is, learning that proceeds without awareness of what is being learned and without intention to learn it-has aroused heated debate in cognitive psychology over the past few decades. In the context of SLA-contrary to Leow (2000), who reported that unawareness does not play an important role in second-language

The study was funded by the Sir Edward Youde Memorial Fellowship awarded to the first author for her doctoral research and was presented at The 28th Annual Conference of the Cognitive Science Society. We are grateful to four anonymous SSLA reviewers for their constructive comments on an earlier version of the article.

*Address correspondence to: Janny Leung, School of English, The University of Hong Kong, Pokfulam, Hong Kong; e-mail: jannyleung@hku.hk. 
(L2) development-Williams (2004, 2005) provided empirical evidence that L2 learning might proceed implicitly. Although there is now some support that language acquisition may draw on implicit learning processes, little is known about the aspects of language that can be learned implicitly and the kind of constraints that act on the implicit learning of language. This study aims to extend Williams's findings.

Traditional studies of implicit learning, or incidental learning for those not concerned with the measurement of awareness, have focused primarily on the implicit abstraction of statistical probabilities in formform connections. It has been shown, for instance, that finite state grammars (Reber, 1967; Reber \& Allen, 1978) can be learned implicitly and that transitional probabilities between syllables as a potential cue to lexical segmentation (Saffran, Newport, \& Aslin, 1996) and phrase structure (Saffran, 2001) can be learned by mere exposure. However, caution should be exercised before generalizing this contention to SLA because language systems, at the core of which lies meaning, are far more sophisticated than simple form-form mappings. Implicit learning of language systems, particularly that of form-meaning connections, warrants independent investigation.

The issue of implicit learning of form-meaning connections amounts to the following: If a person hears a word at the same time that some concept is activated in his or her mind, can this person learn the association between them without any intention of doing so and without any awareness that the word and concept are connected?

The orthodox position seems to be that vocabulary learning-the mapping between content words and lexical meaning-requires explicit processing. Vocabulary learning is believed to depend not just on simple associative learning but also on joint attention (e.g., the sharing, following, and directing of attentional focus between communicative participants) and theory of mind (i.e., the understanding of mental states, as suggested by Bloom, 2000, 2001). Additionally, Krashen and Terrel (1983) linked vocabulary learning to comprehension. Their theory proposed that comprehensibility is "dependent directly on the ability to recognize the meaning of key elements in the utterance" (p. 155).

Amnesics (i.e., people who suffer from memory loss), who show a normal ability in implicit memory tasks, tend to have great difficulty in learning new vocabulary (Gabrieli, Cohen, \& Corkin, 1988; Haist, Musen, \& Squire, 1991; Squire, 1992). Based on the amnesic literature, N. C. Ellis (1994) concluded that attention (but not awareness) is necessary and sufficient for learning novel word forms, whereas the acquisition of word meanings requires both attention and awareness. From a computational perspective, Gupta and Dell (1999) also argued that form-meaning mappings are too arbitrary in nature to be assimilated using the kinds of implicit learning mechanisms that underlie skill learning. 
However, these arguments have been developed almost exclusively in the context of learning words for objects. When it comes to learning associations between grammatical forms and meanings, the situation may be very different. Bloom (2000) acknowledged that meanings of grammatical words (e.g., the article the and the preposition of are acquired through abstraction across memories of instances of use. Indeed, there is no reason why the case of learning words for objects should be any different. As noted by Quine, the learner has no way of knowing which aspect of an object (e.g., a rabbit) is being referred to by a novel word (e.g., referred to as a gavagai by Quine, 1960). Meanings of both lexical and grammatical morphemes may only emerge through abstraction over multiple instances in memory. A few recent clinical studies have shown that amnesics can in fact learn novel associations, like in sequence learning (Gabrieli, Keane, Zarella, \& Poldrack, 1997) or in learning the vocabulary and grammar of a L2 (Hirst, Phelps, Johnson, \& Volpe, 1988). Vargha-Khadem, Gadian, Watkins, Connelly, Van Paesschen, and Mishkin (1997) found normal vocabulary, reading development, and general knowledge in three adult amnesics who had great difficulty recalling events. These results went against the claim that vocabulary learning is necessarily dependent on declarative memory for specific episodes of word use. Rather, meaning may be implicitly abstracted from experience. It is possible that attention and explicit processing are only required for storing the associations between linguistic forms and their situation of use at the moment they are encountered and that the abstraction of meaning over time takes place unconsciously.

The competition model (Bates \& MacWhinney, 1989) provides a framework for thinking about the implicit acquisition of form-meaning connections. Learners track the probabilities with which input forms (e.g., preverbal positioning, verb agreement morphology, sentence initial positioning, nominative case-marking, etc.) are associated with specific meanings (e.g., agency). Such probability tracking is clearly implicitly processed. There is no awareness of these probabilities or of the process by which they are constantly updated through usage. Basic principles of associative learning, such as overshadowing and blocking, can be applied to learning these form-function mappings and can be used to explain first-language (L1) and L2 learning phenomena such as morpheme acquisition orders, fossilization, transfer, and interference (N. C. Ellis, 2006). Implicit learning mechanisms might play an important role in tracking the contingencies between forms and meanings.

Laboratory-type SLA research in implicit learning is mostly restricted to studies that contrasted the learning effect obtained between an explicit condition (rule-search or explicit instruction) and an implicit condition (memorization only; DeKeyser, 1995; Robinson, 1996, 1997) or to studies between participants with a differential level of awareness of the target structure (Alanen, 1995; Leow, 2000; Rosa \& Leow, 2004; Rosa \& 
O'Neill, 1999), without directly testing the notion of whether implicit learning of the target structure is possible. Although these studies typically found that participants who performed a more explicit task or had a higher degree of awareness showed better performance on an experimental task, it may well be that these participants possessed both implicit and explicit knowledge of the target structure, which would fully explain why their performance was better than participants with implicit knowledge only. Moreover, few attempts were made in these studies to provide an optimal condition for implicit learning. Attention was rarely drawn to the relevant form and meaning in the implicit training tasks used. Additionally, training and testing tasks were often distinct, despite the understanding that implicit knowledge is relatively inflexible and context dependent.

Some evidence of implicit learning of grammatical form-meaning connections was found in Williams (2004, 2005). For example, in Experiment 1 of Williams (2005), participants first learned four determinerlike words that functioned similarly to the English definite article, except that they also encoded the distance between the speaker and the object (gi and $r o$ for near objects and $u l$ and ne for far objects). What participants were not told was that the use of these determiners also depended on the animacy of the accompanying noun (gi and $u l$ for animate objects and ro and ne for inanimates). Participants were then exposed to training sentences that combined English lexis with the novel determiners, as in The researchers studied ul bees from a safe distance. Participants had to repeat the sentences, indicate whether the novel word meant near or far by pressing the appropriate response button, and form a mental image of the situation portrayed by the sentence in anticipation of a memory test on sentence content. Afterward, participants performed a surprise generalization test in which novel contexts were used and they had to choose between two possible determiners, one of which violated the animacy rule-for example, The art collector went to Greece to collect ul/ne vases. For the critical (generalization) items, neither determiner had occurred with that noun during training. Thirty-three out of 44 participants claimed that they were not aware of the relevance of animacy to article usage during training or test phases, yet these unaware participants were slightly above chance in selecting the correct article for generalization items in the test phase. This study provided evidence of implicit learning of grammatical form-meaning connections, without attention being explicitly directed to the relevant aspects of meaning.

An important issue in all implicit learning research is whether the tests of awareness are sensitive enough. In an extension of the Williams (2005) study, Hama and Leow (2010) adopted the same system and training task but asked participants to think aloud during the training and test phases. The training phase protocols revealed no instances of 
noticing the animacy correlation, which confirmed that this training procedure was effective in deflecting participants' attention from the hidden regularity. The testing phase protocols revealed that 8 out of 34 participants were aware of the relevance of animacy, at either the level of noticing or understanding. Two of these 8 failed to report animacy in a postexperiment questionnaire, which was similar to that used by Williams. In contrast, there was also one case of a participant who mentioned animacy in the questionnaire but not in the thinkaloud protocol. Thus, there does not appear to be a large difference in sensitivity between postexperiment debriefings and concurrent think-aloud.

In contrast to the Williams (2005) study, Hama and Leow (2010) found that after removing all 9 of the aware participants, the remainder showed no tendency to choose the article with the correct animacy value in the test phase. However, there are a number of procedural differences between the two studies that might account for this null result, the most notable of which is that Hama and Leow used a four-choicerather than a two-choice-test task. Participants had to focus on the learned distance dimension during the decision process, forcing attention to the sentence context as a factor controlling article selection, potentially deflecting attention from the noun. It should also be noted that Hama and Leow's participants even performed poorly on trained items; that is, they could not discriminate animacy-appropriate and animacy-inappropriate items when the correct choice had actually been heard six times in training. In contrast, in Williams, performance on trained items exceeded that of new items. Hama and Leow's result might therefore reflect a global lack of attention to the noun as a potential controlling factor in the test task, or else a failure to reliably encode article-noun combinations during the training phase. None of Hama and Leow's participants knew languages that encode grammatical gender. Given that in Williams $(2004,2005)$ there was evidence that learning effects were barely above chance for participants who knew no gender languages, it hardly seems surprising that Hama and Leow obtained no learning effect. ${ }^{1}$

In this experiment, the generality of implicit learning of form-meaning connections is explored, and an alternative methodology for examining implicit learning in this domain is also developed. With regard to generality, the experiment sought to establish whether implicit learning could be obtained for a different kind of form-meaning connection. The Williams (2004, 2005) studies involved animacy, which, as a semantic feature of nouns, is directly retrievable from the lexicon. DeKeyser's (1995) grammar involved number and thematic role, which are contextually derived features. Given that DeKeyser failed to find implicit learning effects, it could be that implicit learning is limited to cases in which the relevant features can be directly retrieved from the lexicon. 
For example, to learn that gi is associated with animate objects but ro with inanimates, it would be sufficient to store exemplars of determinernoun combinations in memory, and the relevant generalization would emerge from abstraction over the preexisting, context-independent lexical representations of the nouns involved. In contrast, to learn that the suffix -on marks plurality, it is necessary to abstract over representations of instances of use of the word that contains contextually derived information. If this information is not reliably encoded in the input, then inducing the relevant generalization will be more difficult. Additionally, discovering the meaning features that map onto novel forms would presumably be easier when the search space is limited to lexical features as opposed to the much wider range of potentially relevant contextually derived features. To this end, this experiment investigated whether implicit learning could be obtained in a situation in which determiners were correlated with the thematic role of the accompanying noun, which is information that needs to be derived from the context rather than the lexicon.

The second aim of the study was to develop a novel methodology for examining implicit learning of form-meaning connections that did not involve forced-choice decisions. This was inspired by the serial reaction time methodology traditionally used to study implicit learning as well as by work done in vision research, especially the contextual cueing paradigm (Chun, 2000; Jiang \& Chun, 2003) and the derived attention paradigm of Lambert and colleagues (Lambert 2002; Lambert \& Sumich, 1996). According to Chun (2000), people develop sensitivity to statistical regularities in the stimulus environment, which constrains what to expect and where to look. In a visual search task, participants search for a rotated $T$ among a number of distracting rotated $L s$. Unbeknownst to the participants, displays are repeated such that certain spatial configurations of distracters tend to appear with certain target positions. Response times are significantly shorter on these repeated trials than on control trials, for which the distracter positions are determined randomly. This is called the contextual cueing effect. The fact that participants cannot distinguish repeated arrays from random ones in a subsequent forced-choice recognition task suggests that the learning effect is implicit. Thus, an attended stimulus can pick up correlations with so-called ambient stimuli, which can guide attention to the target, even if those stimuli themselves did not receive focal attention. Lambert (2002), again using speed of detection of the onset of the target stimulus as a measurement of learning, found that learning of the predictive relationship between cues (letters $w$ and $s$ ) and targets (the spatial location of objects) orients participants' attention and called this a derived attention effect. Learning proceeded without awareness; when presented with two statements, one of which described the correct cue-target relationship and the other the incorrect use, participants 
exhibited chance performance at picking the correct statement. Lambert and Sumich (1996) showed that spatial attention can also be directed by abstract attributes like the semantic category (e.g., animacy, as in dog vs. hat) of the cue words, which served as a cue to the target locations of objects. Consistent with these findings, Goujon, Didierjean, and Marmèche (2007) reported that the semantic category of the contextual stimuli (whether digits were odd or even) can act as a cue to target location. Participants implicitly learned the relationship between the cue and the target without being able to report any awareness of the predictiveness of the contexts, or to distinguish trials that respected or violated these predictions.

Language processing research has shown that people also develop sensitivity to the relationship between visual and linguistic information. In the visual world paradigm, Tanenhaus, Spivey-Knowlton, Eberhard, and Sedivy (1995) used eye-movement tracking to reveal that visual context affected the resolution of temporary syntactic ambiguity in audio instructions. They described this as evidence for the "rapid and nearly seamless integration of visual and linguistic information" (p. 1633) and suggested that people try to establish reference during the earliest moments of syntactic processing. Assuming a close link between language forms and attentional processes, changes in attentional processes (i.e., referent identification) are used over time as indicators of learning.

There are three significant improvements to this new methodology, which makes the current experiment potentially more sensitive to implicit learning effects than earlier studies like Williams (2004, 2005) and Hama and Leow (2010). First, a concurrent measure of learning, as opposed to a posttraining grammaticality judgment test, was used. From the participants' point of view, there was no division between training and test phases but rather just one task performed across one continuous stream of trials. This is desirable according to the transferappropriate processing framework, which has postulated that performance on an implicit memory task receives facilitation in proportion to the degree of overlap between study and test processing (Roediger, 1990). In a similar manner, implicit learning in serial reaction time tasks has been shown to be inflexible and context-specific (Jiménez, Vaquero, \& Lupianez, 2006), and therefore, given the limited time for learning in a laboratory setting, task change is best avoided. In SLA, it has also been acknowledged that transfer of knowledge acquired in receptive modalities to the use of knowledge in productive modalities seems weak (Hulstijn, 2002). Second, the training and testing task used involved comprehension rather than production. Because knowledge has to reach a higher threshold to be expressed in production, the lesser processing demand in our task makes it more sensitive to implicit knowledge. Finally, a reaction time task that introduced time pressure was 
used. It was assumed that this encouraged the use of implicit rather than explicit knowledge. Destrebecqz and Cleeremans (2001) found the strongest evidence for implicit learning in a serial reaction time task when there was no delay between a response and the next stimulus. In a SLA study, R. Ellis (2005) found that timed and untimed grammaticality judgment test performance depended on the learners' implicit and explicit knowledge, respectively. R. Ellis (2005) reasoned that this was because time pressure encouraged the use of feel rather than rule and limited the opportunity to access metalinguistic knowledge. Therefore, it is assumed that the time pressure induced by the reaction time test reduces the likelihood that participants will become aware of the target regularity (the mapping between articles and thematic roles) and encourages reliance on implicit knowledge.

\section{THE EXPERIMENT}

\section{Materials}

The current experiment investigates the implicit learning of a mapping between thematic roles and a set of novel determiners. Participants were introduced to four artificial articles: gi, ro, $u l$, and ne, where gi and ro were used before personal names referring to adults and $u l$ and ne were used before personal names referring to children. What participants had not been told was that article use also depended on the thematic role of the accompanying noun phrase; gi and $u l$ were used with agents and ro and ne were used with patients. The terms agent and patient here referred to Dowty's (1991) notions of proto-agent and protopatient, which have a broader definition than the senses they bear in generative grammar. For instance, the category agent would include technical concepts such as agents, actors, causers, and so forth. The miniature article system is presented in Table 1.

Each experimental trial consisted of a distinct picture (like that in Figure 1) with an accompanying audio description, such as Kiss ul Mary a boy on the face. The participants' primary task was to indicate as quickly as possible whether the named individual (Mary in this case;

Table 1. Miniature article system used

\begin{tabular}{lll}
\hline Person & Agent & Patient \\
\hline Adult & $G i$ & Ro \\
Children & $U l$ & $N e$ \\
\hline
\end{tabular}




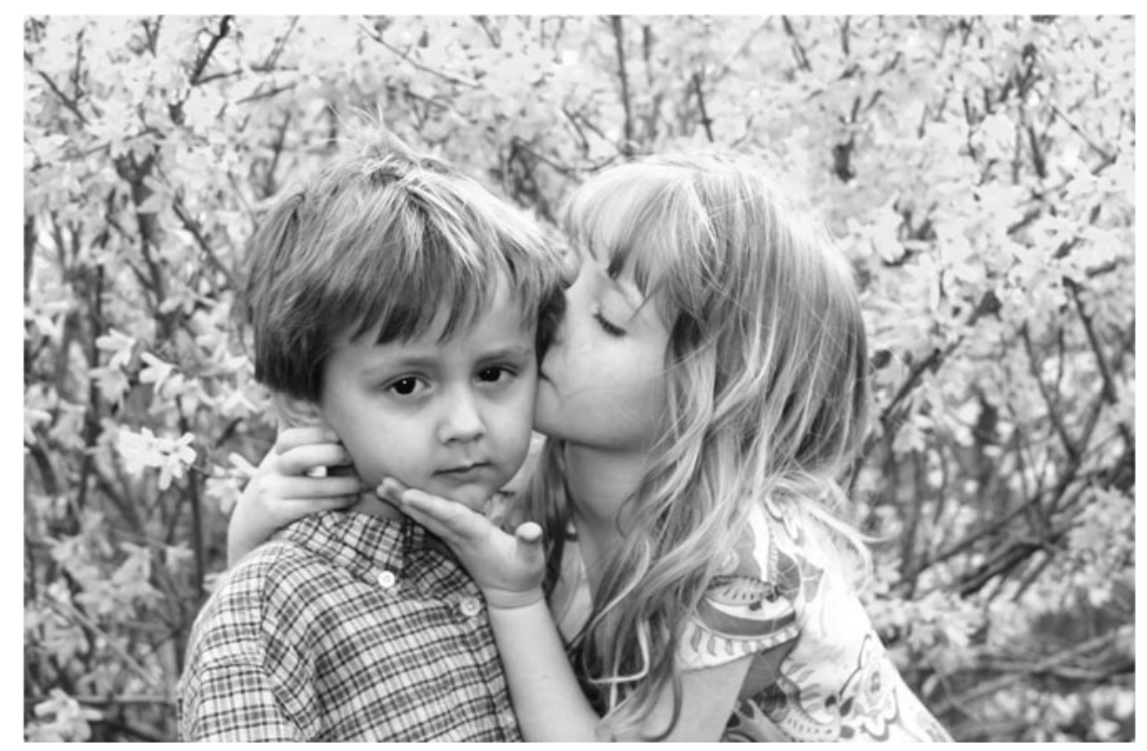

Figure 1. A sample picture.

only one person was named for each picture) appeared on the left- or right-hand side of the picture. To avoid some articles becoming more salient than others due to the position they appeared in, flexible sentence orders were used in the picture descriptions. Sentences included a verb (V), noun phrase 1 (NP1), noun phrase 2 (NP2), and prepositional phrase (PP), following the pattern V + NP1 + NP2 + PP. Whereas either NP1 or NP2 could be an agent or patient, NP1 was always a proper name and NP2 was always a common noun; V was always in its infinitive form. Therefore, the two possible sentence constructions to describe Figure 2 are given in (1) and (2):
(1) Kiss ul Mary
a boy
$\mathrm{V}$ NP1(Agent) NP2 (Patient)
on the face.
(2) Kiss
$\mathrm{V}$
$\begin{array}{ll}\text { ne David } & \text { a girl } \\ \text { NP1(Patient) NP2 (Agent) }\end{array}$
$\mathrm{PP}$
on the face.
PP

Verb-subject-object (VSO) and verb-object-subject (VOS) structures exist in natural languages. For example, VSO is the dominant word order in Samoan, but VOS is also possible and common (Robinson, 2002).

Three common names for each gender were used with each possible article with equal frequency to avoid any input bias in article-name associations. The frequency of agents and patients appearing as NP1 was balanced. 
Because word order does not reveal the thematic roles of nouns, the determiners are the only surface forms that mark this meaning. If participants learned the correlation between articles and thematic roles, then their attention could, in principle, be directed to the appropriate individual in the picture even before hearing the proper name, thereby facilitating their responses (e.g., on hearing Kiss $u l$. . they would know that the named individual was the agent of the action and therefore choose the girl on the right of the picture). It should also be the case that if the mapping between articles and thematic roles is then reversed (in what will be referred to as the violation block), response times should be slowed and errors should increase. If the mapping between articles and thematic roles is learned implicitly, then these effects should be obtained even if the participants have no awareness that there is an association between articles and thematic roles or that it was reversed in the violation block.

One hundred twenty pictures were adopted so that an agent and a patient of the depicted action were clearly identifiable. Eighty-eight of these were used for the training phase and 32 were used for the testing phase. The training phase items were devised so that participants could use a variety of cues to determine their response apart from the article-thematic role mappings (which could determine the correct response in all cases). Fifteen of the pictures involved interactions between an adult and child. Given that the participants were told that two of the articles were used with children and two with adults, it would be possible, in principle, for responses to these items to be based solely on the form of the article. These items were important in orienting participants' attention to the articles. For 65 training items, the depicted individuals were both adults or children but different in gender. In such cases, participants would be likely to respond on hearing the proper name. For eight items, both individuals depicted were of the same age and gender. In such cases, participants would presumably rely on cues later in the sentence to make their response (assuming no knowledge of the target system). For example, when there were two men in the picture and the sentence was Arrest ro David a police officer, participants were able to distinguish David based on the fact that the man in the police uniform was the unnamed individual. In this case, participants may have reacted after hearing further cues that did not come until after the proper name. The variety of cue types was designed to motivate and habituate the participants to respond at different points of hearing the audio presentation, using as little information as possible.

The critical data came from the last 32 trials of the experiment, which were divided into control and violation blocks. There was no division between these trials and the training from the participant's point of view. All of the pictures used in these blocks depicted either 
two adults or two children. For this reason, the articles could have predictive value only if participants knew the correct mapping from articles to thematic roles. In the control block, the sentences respected the same system used in training, whereas in the violation block, the mapping between articles and thematic roles was reversed so that gi and $u l$ were used with patients and ro and ne were used with agents. Items were rotated between the control and violation blocks over two groups of randomly assigned participants so as to control for differences in item difficulty. For example, one group of participants received the correct Examine gi Mary a patient in a dental clinic in the control block, whereas the second group received that item in the violation block in the incorrect form Examine ro Mary a patient in a dental clinic.

The item and cue types are summarized in Table 2. Note that in all cases, if participants had learned the article-thematic role mapping, they would be able to base their decisions solely on the articles. Even if knowledge of this mapping remained implicit, it is possible that it would facilitate responses that the participant believed to be based on the other available cues.

All aural stimuli were recorded by the second author, a native speaker of British English. The interval between the onset of the article and onset of the name was exactly $1 \mathrm{~s}$, and the duration of the article was $0.5 \mathrm{~s}$. This provided a time window in which the article could be used to orient attention even before the name was presented. The time to indicate whether the named individual was on the right or the left of the picture was measured from the onset of the article.

All training trials were randomized individually for each participant, as were all trials within the control and the violation blocks.

Table 2. Distribution of cue types in the training and testing phase

\begin{tabular}{|c|c|c|c|}
\hline Cue types & Cued by & $\begin{array}{c}\text { Percentage } \\
\text { of items }\end{array}$ & $\begin{array}{l}\text { Number } \\
\text { of items }\end{array}$ \\
\hline \multicolumn{4}{|l|}{ Training } \\
\hline Different gender & The proper name & $73.9 \%$ & 65 \\
\hline Different age & The determiner & $17 \%$ & 15 \\
\hline Other cues & $\begin{array}{l}\text { Words that appear after } \\
\text { the proper name }\end{array}$ & $9.1 \%$ & 8 \\
\hline Total & & $100 \%$ & 88 \\
\hline \multicolumn{4}{|c|}{ Testing (control and violation) } \\
\hline Different gender & The proper name & $100 \%$ & 32 \\
\hline Total & & $100 \%$ & 32 \\
\hline
\end{tabular}




\section{Procedure}

The participants filled out a language background questionnaire to ensure that they had no advanced knowledge of languages with highly developed case systems such as German or Latin. They then received vocabulary pretraining for the four articles. They were told that the order of words in the sentences presented to them would not be as in English. Examples were given so that participants became familiar with the articles and the sentence structure. They were given as much time as they needed for this pretraining. Nine sample pictures were provided in the instructions to help familiarize the participants with the adultchild distinction in the determiners.

All procedures of the experiment were computerized and standardized. The experimenter was available to clarify any questions when needed. Superlab for Windows Pro 2.0 was used to provide all of the pretraining, instructions, stimulus presentation, and data collection (i.e., reaction time measurement). All verbal responses were recorded on an audiotape. A two-button serial mouse was used as the input device. Participants were told to rest their left and right index fingers on the buttons.

The experiment was disguised as a reaction time test, and the participants had to click to proceed from one task to the next. For each picture, the participants had to perform three tasks.

Picture Description. The participants were asked to describe the picture in their own words. This task was used to ensure that participants paid attention to the action and the direction of action as shown on the picture.

Reaction Time Test. The participants had to click left or right on the mouse as quickly as possible as they listened to an audio picture description, as soon as they could decide on which side of the picture the named individual appeared. Responses had to be made before the end of the auditory sentence (responses beyond this limit were not recorded). If participants gave a wrong answer, the page would reload and they would have to start the audio presentation and reaction time test again. Reaction times for second and further attempts were excluded from the data analysis. This task was the crucial measurement of the experiment.

Sentence Reformulation. This task was designed so that the participants put the sentence they heard into correct English order, retaining the article used (to stimulate concurrent active processing of the action portrayed and the article used). The participants were not constrained in their choice of syntactic structure in their reformulations. For example, the sentence Examine gi Mary a patient in a dental clinic could be reformulated as Gi Mary examined a patient in a dental clinic or A patient was examined by gi Mary in a dental clinic. The task forced the participants to concurrently process the form (articles) and the related meaning (thematic roles). 
A recall test was inserted between every two pictures so that participants would repeat the sentences they had reformulated in correct English order for the last two pictures. During this recall test, the participants were able to look at the pictures again, with a prompt that read "???" to remind them of the reformulation task. Participants were told that exact wording was not needed but that it was important to use the exact articles used. Feedback was not provided for this task, but participants knew that their answers had been recorded. The recall test ascertained that participants listened to the whole sentence in the audio presentation to make sure that they interpreted the action in the picture (and thus the roles of the individuals involved) the way it was intended. Participants were also encouraged to rely on the pictures as cues to the recall task. By doing so, it was hoped that the form and meaning of the articles would be activated concurrently when the participants tried to memorize and recall the sentences.

Participants were reminded before the start of the experiment that they might be able to react more quickly if they paid attention to the articles. This was the case even assuming no knowledge of the mapping between articles and thematic roles because they had learned that gi and ro were only used with adults and $u l$ and ne were only used with children.

An interview was conducted at the end of the experiment. Participants were asked if they had any feelings about when gi versus ro and $u l$ versus ne were used. They then looked through some of the training items again (no more than 10 items) and were asked to report any idea that came to mind. They were encouraged to make as many guesses as possible. It was assumed by the authors that instruction to guess makes the procedure sensitive even to conscious knowledge in which the participants have little confidence. There was therefore not just reliance on responses to open-ended questions whose sensitivity to conscious knowledge has been questioned (Shanks \& St. John, 1994) but also on the guesses that are produced when prompted by further training trials. Participants were classified as being aware or unaware on the basis of their reported knowledge of the target form-meaning mapping.

The whole experiment took approximately $1 \mathrm{hr}$ to complete. A 3-min break was inserted in the middle of the experiment.

\section{Participants}

Twenty-five native English-speaking undergraduate and graduate students at the University of Cambridge participated in this study. Eleven of them studied Arts subjects, five studied Social Sciences, and nine studied Science or Engineering. No participant had advanced knowledge of any languages with highly developed case systems, such as German or Latin. Foreign languages known to the participants were French $(n=14)$, 
Spanish $(n=10)$, Latin $(n=2$ intermediate), Bengali $(n=1)$, Chinese $(n=1)$, German $(n=1$ intermediate), Hindi $(n=1)$, Irish $(n=1)$, Italian $(n=1)$, Persian ( $n=1)$, and Russian ( $n=1$ intermediate). Participants were semirandomly assigned to the two presentation lists formed by rotating items around the control and violation blocks such that, ultimately, there were equal numbers of unaware participants on each list.

\section{RESULTS}

\section{Postexperiment Report}

Twenty out of 25 participants (80\%) remained unaware of the system at the end of the experiment. Many unaware participants said it did not come to their mind at all that the two articles used for adults and children were used in different conditions and were surprised when asked to comment on it. Five thought during the experiment that there might be a differentiation on the basis of gender, but after some trials, they realized that this was not the case. A further 6 participants mentioned gender when invited to make guesses during the debriefing. ${ }^{2}$

The remaining 5 participants who were able to link the use of articles with concepts such as someone doing something to the other person or one person takes an active role and the other is more passive were all counted as aware, even if these were not the first guesses they made. One of the aware participants reported only becoming aware of the possible relevance of thematic role after the experiment. The remaining 4 said that they had become aware during the experiment, but 2 of them said that that they had not had a chance to verify it. All of these participants were classified as aware to make sure that participants who had an awareness of the implicit system but had low confidence in the explicit knowledge were not included in the unaware data, which is the focus of the analysis. Among the 3 participants with some knowledge of German, Russian, or Latin, 2 were aware participants (1 with intermediate knowledge of Latin and Russian and 1 with intermediate German). The other participant who had intermediate Latin was unaware.

\section{Unaware Participants}

Outlying response times were identified by setting cutoff limits at \pm 2.5 standard deviations from each participant's mean in the control and violation blocks, respectively. Outlying values were replaced with the appropriate cutoff value. Figure 2 shows the average reaction time for correct selection of the position of the named individual against trial 


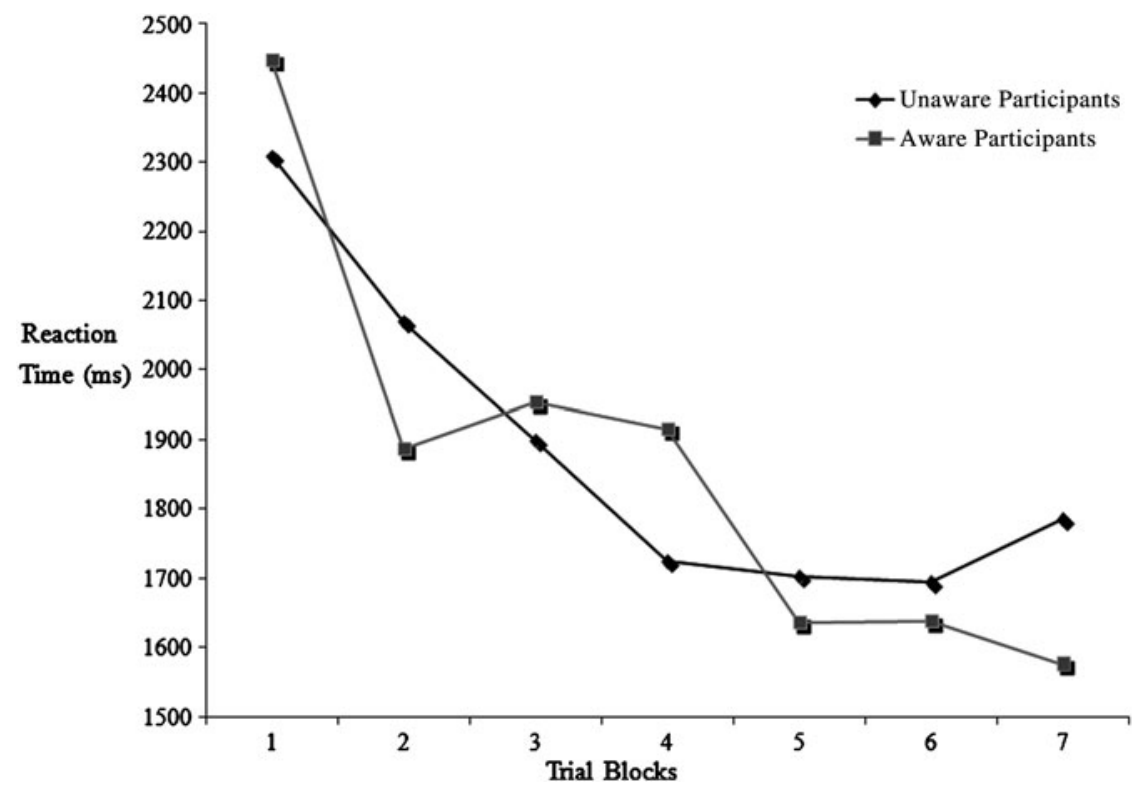

Figure 2. Reaction times across blocks for aware and unaware participants.

blocks in different gender items for both the aware and the unaware participants.

Each block comprises 16 trials (apart from the first 8, which were for task familiarization). The control block is block 6 , and the violation block is block 7 . With respect to the unaware participants, there is clearly a general trend for reaction times to decrease throughout the experiment, especially over the first four blocks. However, there was a sudden increase in reaction times at the violation block. The mean reaction time in the control block was $1694 \mathrm{~ms}, S E=40 \mathrm{~ms}$, and in the violation block, it was $1785 \mathrm{~ms}, S E=43 \mathrm{~ms}$. A repeated-measures ANOVA showed that the reaction times for these blocks were significantly different, $F(1,19)=4.64, p<.05$, partial $\eta^{2}=.196 .^{3}$ As a more fine-grained measure of the violation effect, the reaction times in the last eight items in the control block were compared with the first eight items in the violation block. The reaction times were $1673 \mathrm{~ms}, S E=49 \mathrm{~ms}$, and $1796 \mathrm{~ms}, S E=51 \mathrm{~ms}$, respectively, and they were also significantly different, $F(1,19)=4.97, p<.05$, partial $\eta^{2}=.207$. However, it is important to mention that, for this comparison, the items were not matched because of the randomized presentation order within the entire control/ violation block. Moreover, there were a few attempts among the unaware participants to respond in under $1 \mathrm{~s}$ (i.e., before they heard the proper names), providing further evidence that they directed their 
attention to the appropriate individual using the thematic role information in the articles.

There was no significant increase in errors in the violation block, the percentage of errors being $3.1 \%$ in the control block and $3.4 \%$ in the violation block, $F(1,19)=0.05, p=.75$. There was not a significant difference in the proportion of outliers either, which were $3.4 \%$ and $3.1 \%$ in the control and violation blocks, respectively, $F(1,19)=0.11, p=.62$. The proportion of failures to respond before the end of the sentence increased from $1.6 \%$ in the control block to $3.4 \%$ in the violation block, but this difference was not significant, $F(1,19)=3.35, p=.08, \eta^{2}=.15$.

Error rate in the repetition ( $94.6 \%$ correct) and recall (86.3\% correct) tasks remained rather stable throughout the experiment.

\section{Aware Participants}

Given that there were only five aware participants, any analysis of their performance must be extremely tentative. However, the reaction times for the aware group did appear to show a rather different pattern over the course of the experiment compared to the unaware group. Although there was a rapid decrease in reaction times at the beginning of the experiment, the average reaction time flattened out before falling again after block 4 . There was no significant difference between the control and violation blocks; the mean reaction times were $1592 \mathrm{~ms}, S E=172 \mathrm{~ms}$, and $1497 \mathrm{~ms}, S E=113 \mathrm{~ms}$, respectively, $F(1,4)=1.71, p=.26$. The proportion of errors and outliers did not differ between the control and violation blocks, which were $11.2 \%$ and $10 \%$, respectively, for errors, and $2.5 \%$ and $3.8 \%$, respectively, for outliers. There were rather more failures to respond before the end of the sentence in the violation block, $18.8 \%$, than the control block, $5 \%$, but this difference was not significant, $F(1,4)=3.14, p>.1$. It should be noted that the overall error rate was higher for the aware than the unaware participants, which were $10.6 \%$ and $3.3 \%$, respectively, $F(1,23)=8.74, p<.01$, partial $\eta^{2}=.275$.

\section{DISCUSSION}

Twenty out of the 25 participants in the experiment claimed to have no awareness of the mapping between the articles and thematic roles. However, when this mapping was reversed in the final block of the experiment, their reaction times increased significantly compared to the preceding control block. It is therefore concluded that they had implicitly learned that certain articles were associated with certain thematic roles, which supports the hypothesis that form-meaning connections 
can be learned implicitly. In previous research, such effects have only been obtained for animacy, a lexical property of nouns (Lambert \& Sumich, 1996; Williams, 2004, 2005). The results of the current study suggest that contextually derived form-meaning connections might also be implicitly learned.

However, what might be the actual cause of the slowdown in the violation block? One explanation follows the logic of the contextual paradigm (Jiang \& Chun, 2003) that inspired the present methodology. In those experiments, participants were able to make use of cues to locate the target more quickly, and their reaction times were affected when this critical association was interrupted. This occurred even though participants appeared to be unaware that the cues were predictive of target location. In the present case it is possible to argue that, for example, on hearing the article gi, participants would direct their attention to the agent of the action (without knowing why) and expect the name of the corresponding individual. In the violation block their anticipation would be wrong (in this example they would hear the name of the patient instead), which would cause slower responses or a trend toward more response failures. There are two problems with this interpretation, however. First, there is no direct evidence that participants do actually direct their attention to the individual cued by the article. Perhaps with the use of eye-movement tracking it would be revealed whether a participant's gaze is misdirected on violation trials. We are currently conducting experiments to explore this possibility. Second, there was no increase in errors in the violation block, yet an attentional explanation would appear to predict that there should have been. This might suggest that the violation effect is in fact caused by what is essentially an agreement violation. The named individual is recognized as being either the agent or the patient of the action, and this information conflicts with the thematic role that is implicitly cued by the article. Such a mismatch would not necessarily provoke a response error because the conflict is irrelevant to the decision as to whether the named individual is on the left or right of the screen. It is important to stress, however, that whether the violation effect is caused by misdirected attention or an agreement violation does not affect in any way the central claim that the effect occurs because participants have learned the mapping between articles and thematic roles.

Although the small number of aware participants $(n=5)$ makes it impossible to make strong claims about their behavior, there is some evidence that they showed a rather different pattern of results from the unaware group. Although their reaction times showed a sharp decrease early in training, presumably reflecting increasing fluency at performing the task, they later rose before gradually decreasing again. It is likely that these participants began forming and testing hypotheses and this caused a delay in their responses. The figuring-out process took place at different stages for each individual, leading to irregularity in the 
overall pattern. It is surprising, however, that there was no significant difference in reaction times between the control and the violation blocks. It should be noted that due to the awareness criterion used, the aware participants had different degrees of awareness, which contributed to the variability of the data. The higher rate of errors and response failures in the aware group compared to the unaware group might have reflected hypothesis formation and testing among the semiaware participants. Because of the small sample size of the aware group, there is no attempt to make any claims regarding their behavior here. It might be useful to consider the results of Lambert (2002), who also reported a lack of a learning effect in semiaware participants, which he interpreted as attributable to the formation and testing of incorrect hypotheses concerning the cuetarget relation, leading to inappropriate orienting toward an invalid location. Likewise, Frensch et al. (2002) reported an increase in reaction time variance before an observed learning effect was evident. Schmidt (2001) also noted that unconsciously perceived stimuli lead to automatic reactions, but consciously perceived stimuli allow participants to modify their reactions. Further research is needed to ascertain the precise behavioral pattern of participants with a varying degree of explicit knowledge.

The comparison of the aware and unaware groups is at least suggestive of differences in performance, and this provides encouraging converging evidence for the classification of unaware and aware participants based on verbal report. There is also evidence of a correspondence between verbal report and reaction time task performance in a related study (Leung \& Williams, 2010) that, using a similar methodology, examined implicit learning of a mapping between novel articles and animacy (as in Williams, 2005). In this task, participants were presented with two pictures-for example, an armchair on the left and a goldfish on the right-and heard a single noun phrase, gi goldfish. Their task was to indicate whether the phrase referred to an animate or inanimate object by pressing one of two response keys. They were told that gi and $u l$ are used with near objects and ro and ne were used with far objects (distance was represented by screen size and position of the object). What they were not told was that gi and ro were used with animate objects and $u l$ and ne were used with inanimates. Learning of the animacy correlation was evaluated by comparing control and violation blocks at the end of the experiment. Out of 33 participants, a total of 20 appeared not to have been aware of the correlation between animacy and article use as assessed by the same debriefing procedure used here. Yet these participants showed a significant slowdown in response times in the violation block, as in this study, which provides further evidence of learning a form-meaning mapping without awareness (although one that is lexically, rather than contextually, derived). Among the 13 aware participants, there were interesting correspondences between verbal report and reaction time performance. Ten participants 
claimed to have become aware of the system before the control block. Not surprisingly, they showed a large-and significant-violation effect. In fact, their mean response time in the control block was actually less than $1 \mathrm{~s}$, meaning that they were able to base their decisions on the article before hearing the noun (all control and violation block trials used pictures of the same distance, such that fast responses must be based on animacy information). This shows evidence of strategic use of conscious knowledge, something that was not possible in the present experiment because the instruction was to respond according to the named individual. It is necessary to stress that not a single response time of under $1 \mathrm{~s}$ was found in the control block data of the unaware group. There was no evidence of participants not reporting awareness of animacy, but using it strategically in the reaction time task. Additionally, the verbal report of the remaining three aware participants suggested that they only became aware of the animacy correlation toward the end of the experiment. They showed no response times of less than $1 \mathrm{~s}$ and no slowdown in the violation block-in fact, they were slightly faster than in the control block, as here, which suggests that they had either learned the violation block mapping or were still in the process of working it out. There was thus an encouraging correspondence between the verbal reports and actual reaction time task behavior. This confirmed that the procedure for assessing awareness is sufficiently sensitive.

The findings from these studies seem to run counter to the idea that the acquisition of meaning depends on explicit processes and more generally to the pervasive idea that awareness is necessary for learning. Within psychology, it has been argued that not even the most basic form of learning-classical conditioning-can be obtained without awareness of the association between the conditioned and unconditioned stimuli-for example, the tone and puff of air in an eye-blink conditioning procedure (Lovibond \& Shanks, 2002, but see Manns, Clark, \& Squire, 2002, for counter evidence). Within SLA research, the necessary connection between awareness and learning has long been noted (Schmidt, 1990), and empirical investigations of L2 learning have found no evidence of learning without awareness (e.g., Leow, 2000). However, there is not necessarily a contradiction between these claims and the present findings. This is because it was assumed that in this study, the participants were fully aware of (i.e., they noticed) the critical phrase (e.g., ul Mary) and its association to the entity (e.g., a girl) within the event with which it was paired (e.g., a girl kissing a boy). In this sense, the participants were aware of a form-meaning relationship at the moment of encoding. What they were not aware of was the specific relationship between the article and the thematic role of that entity being implicit within their understanding of the event. It is assumed that this specific relationship was learned implicitly through unconscious abstraction processes operating over representations of multiple 
training events in memory. This could be regarded as a form of crosssituational statistical learning of the kind that has been found to guide acquisition of the meanings of content words in adults and infants (Smith \& Yu, 2008). When the relationship between a form and a complex event is ambiguous, it can be inferred by making comparisons across different events, even when the events are separated in time. In a sense, although awareness of the relevant forms and events is clearly necessary for encoding them in memory, subsequent abstraction processes may lead to generalizations of which the learner is not aware.

It is important to note that this study has only shown that implicit learning influences a receptive task-and for that matter, a task that is identical to that in which learning occurred. It remains an open question whether transfer to different tasks, and, in particular, spontaneous production tasks, requires awareness of the relevant form-meaning mappings. Nevertheless, the learning process that has been isolated in this study is of very wide generality. It can pick up correlations with meaning features that are implicit in lexical representations of word meaning (e.g., animacy) or implicit in representations of events (e.g., thematic role). It could thus underlie naturalistic acquisition of grammatical form-meaning connections in the domains of, for example, definiteness or tense and aspectual distinctions. It could also provide a mechanism for fine-tuning meanings of open-class words over successive instances of use. However, this mechanism is not necessarily so general that it is entirely unconstrained. Just as Quine's (1960) gavagai problem of indeterminate reference calls for meaning induction to be constrained by heuristic strategies, so too the search space for grammatical form-meaning connections may need to be delimited by prior assumptions about potentially encodable distinctions (Bickerton, 1999, 2001). In the case of SLA, the L1 may bias selection of forms for encoding in memory through mechanisms of learned attention (N. C. Ellis, 2006) or may provide the inventory of meaning features that are available for association with those forms. Therefore, even though it is claimed here that implicit learning of form-meaning connections has wide generality, the degree to which it is constrained awaits future investigation.

\section{(Received 4 February 2010)}

\section{NOTES}

1. In Williams (2005, Experiment 1), the seven participants who knew no gender languages were only $54 \%$ correct on generalization items (not significantly different from chance). Nevertheless, their performance on trained items was $74 \%$, which was significantly different from chance $(p<.001)$.

2 . Note that our use of the term aware refers specifically to being aware of the relevance of thematic role. Including participants who are aware of other, irrelevant, factors, 
such as gender in the unaware group is actually a conservative strategy because it could reduce sensitivity to effects of thematic role.

3. An ANOVA was run with List as a between-participants factor. Because there was no main effect of list, $F(1,18)=1.01, p=.33$, and more importantly, absolutely no evidence of an interaction between list and violation effect, $F(1,18)=0.02, p=.88$, this factor was removed from further analyses.

\section{REFERENCES}

Alanen, R. (1995). Input enhancement and rule presentation in second language acquisition. In R. Schmidt (Ed.), Attention and awareness in foreign language learning and teaching (pp. 259-302). Honolulu: University of Hawai'i Press.

Bates, E., \& MacWhinney, B. (1989). Functionalism and the competition model. In B. MacWhinney \& E. Bates (Eds.), The crosslinguistic study of sentence processing (pp. 3-73). New York: Cambridge University Press.

Bickerton, D. (1999). Creole languages, the language bioprogram hypothesis, and language acquisition. In W. C. Ritchie \& T. K. Bhatia (Eds.), Handbook of child language acquisition (pp. 195-220). San Diego, CA: Academic Press.

Bickerton, D. (2001). Okay for content words, but what about functional items? Commentary on Bloom: How children learn the meanings of words. Behavioral and Brain Sciences, 24, 1104-1105.

Bloom, P. (2000). How children learn the meanings of words. Cambridge, MA: MIT Press.

Bloom, P. (2001). Précis of how children learn the meanings of words. Behavioral and Brain Sciences, 24, 1095-1103.

Chun, M. M. (2000). Contextual cueing of visual attention. Trends in Cognitive Sciences, 4, $170-178$.

DeKeyser, R. M. (1995). Learning second language grammar rules: An experiment with a miniature linguistic system. Studies in Second Language Acquisition, 17, 379-410.

Destrebecqz, A., \& Cleeremans, A. (2001). Can sequence learning be implicit? New evidence with the process dissociation procedure. Psychonomic Bulletin and Review, 8 , $343-350$.

Dowty, D. (1991). Thematic proto-roles and argument selection. Language, 67, 547-619.

Ellis, N. C. (1994). Vocabulary acquisition: The implicit ins and outs of explicit cognitive mediation. In N. C. Ellis (Ed.), Implicit and explicit learning of languages (pp. 211-282). London: Academic Press.

Ellis, N. C. (2006). Selective attention and transfer phenomena in L2 acquisition: Contingency, cue competition, salience, interference, overshadowing, blocking, and perceptual learning. Applied Linguistics, 27, 164-194.

Ellis, R. (2005). Measuring implicit and explicit knowledge of a second language: A psychometric study. Studies in Second Language Acquisition, 27, 141-172.

Frensch, P. A., Haider, H., Runger, D., Neugebauer, U., Voigt, S., \& Werg, J. (2002). Verbal report of incidentally experienced environmental regularity: The route from implicit learning to verbal expression of what has been learned. In L. Jiménez (Ed.), Attention and implicit learning (pp. 335-366). Amsterdam: Benjamins.

Gabrieli, J. D. E., Cohen, N. J., \& Corkin, S. (1988). The impaired learning of semantic knowledge following medial temporal lobe resection. Brain and Cognition, 7, 157-177.

Gabrieli, J. D. E., Keane, M., Zarella, M. M., \& Poldrack, R. A. (1997). Preservation of implicit memory for new associations in global amnesia. Psychological Science, 8, 326-329.

Goujon, A., Didierjean, A., \& Marmèche, E. (2007). Contextual cueing based on specific and categorical properties of the environment. Visual Cognition, 15, 257-275.

Gupta, P., \& Dell, G. S. (1999). The emergence of language from serial order and procedural memory. In B. MacWhinney (Ed.), The emergence of language (pp. 447-481). Mahwah, NJ: Erlbaum.

Haist, F., Musen, G., \& Squire, L. (1991). Intact priming of words and nonwords in amnesia. Psychobiology, 19, 275-285.

Hama, M., \& Leow, R. P. (2010). Learning without awareness revisited: Extending Williams (2005). Studies in Second Language Acquisition, 32, 465-491.

Hirst, W., Phelps, E. A., Johnson, M. K., \& Volpe, B. T. (1988). Amnesia and second language learning. Brain and Cognition, 8, 105-116. 
Hulstijn, J. H. (2002). Towards a unified account of the representation, processing and acquisition of second language knowledge. Second Language Research, 18, 193-223.

Jiang, Y., \& Chun, M. M. (2003). Contextual cueing: Reciprocal influences between attention and implicit learning. In L. Jiménez (Ed.), Attention and implicit learning (pp. 277-296). Amsterdam: Benjamins.

Jiménez, L., Vaquero, J. M. K., \& Lupianez, J. (2006). Qualitative differences between implicit and explicit sequence learning. Journal of Experimental Psychology: Learning, Memory, and Cognition, 32, 475-490.

Krashen, S. D., \& Terrell, T. D. (1983). The natural approach: Language acquisition in the classroom. Englewood Cliffs, NJ: Prentice Hall.

Lambert, A. J., \& Sumich, A. L. (1996). Spatial orienting controlled without awareness: A semantically-based implicit learning effect. Quarterly Journal of Experimental Psychology, 49, 490-518.

Lambert, T. (2002). Visual orienting, learning and conscious awareness. In L. Jiménez (Ed.), Attention and implicit learning (pp. 253-275). Amsterdam: Benjamins.

Leow, R. P. (2000). A study of the role of awareness in foreign language behavior: Aware versus unaware learners. Studies in Second Language Acquisition, 22, 557-584.

Leung, J., \& Williams, J. N. (2010). Constraints on implicit learning of grammatical formmeaning connections. Manuscript submitted for publication.

Lovibond, P. F., \& Shanks, D. R. (2002). The role of awareness in Pavlovian conditioning: Empirical evidence and theoretical implications. Journal of Experimental Psychology: Animal Behavior Processes, 28, 3-26.

Manns, J. R., Clark, R. E., \& Squire, L. R. (2002). Standard delay eye blink classical conditioning is independent of awareness. Journal of Experimental Psychology: Animal Behavior Processes, 28, 32-37.

Quine, W. V. O. (1960). Word and object. Cambridge, MA: MIT Press.

Reber, A. S. (1967). Implicit learning of artificial grammars. Journal of Verbal Learning and Verbal Behavior, 6, 855-863.

Reber, A. S., \& Allen, R. (1978). Analogic and abstraction strategies in synthetic grammar learning: A functionalist interpretation. Cognition, 6, 189-221.

Robinson, P. (1996). Learning simple and complex second language rules under implicit, incidental, rule-search, and instructed conditions. Studies in Second Language Acquisition, $18,27-67$.

Robinson, P. (1997). Individual differences and the fundamental similarity of implicit and explicit adult second language learning. Language Learning, 47, 45-99.

Robinson, P. (2002). Effects of individual differences in intelligence, aptitude and working memory on adult incidental SLA: A replication and extension of Reber, Walkenfeld and Hernstadt, 1991. In Peter Robinson (Ed.), Individual differences and instructed language learning (pp. 211-266). Amsterdam: Benjamins.

Roediger, H. L. (1990). Implicit memory: Retention without remembering. American Psychologist, 45, 1043-1056.

Rosa, E. M., \& Leow, R. P. (2004). Awareness, different learning conditions, and second language development. Applied Psycholinguistics, 25, 269-292.

Rosa, E., \& O'Neill, M. D. (1999). Explicitness, intake, and the issue of awareness. Studies in Second Language Acquisition, 21, 511-556.

Saffran, J. R. (2001). The use of predictive dependencies in language learning. Journal of Memory and Language, 44, 493-515.

Saffran, J. R., Newport, E. L., \& Aslin, R. N. (1996). Word segmentation: The role of distributional cues. Journal of Memory and Language, 35, 606-621.

Schmidt, R. (1990). The role of consciousness in second language learning. Applied Linguistics, 11, 129-158.

Schmidt, R. (2001) Attention. In P. Robinson (Ed.), Cognition and second language instruction (pp. 3-32). New York: Cambridge University Press.

Shanks, D. R., \& St. John, M. F. (1994). Characteristics of dissociable human learning systems. Behavioral and Brain Sciences, 17, 367-447.

Smith, L., \& Yu, C. (2008). Infants rapidly learn word-referent mappings via cross-situational statistics. Cognition, 106, 1558-1568.

Squire, L. (1992). Memory and the hippocampus: A synthesis from findings with rats, monkeys, and humans. Psychological Review, 99, 195-231. 
Tanenhaus, M. K., Spivey-Knowlton, M., Eberhard, K., \& Sedivy, J. (1995). Integration of visual and linguistic information during spoken language comprehension. Science, $268,1632-1634$.

Vargha-Khadem, F., Gadian, D. G., Watkins, K. E., Connelly, A., Van Paesschen, W., \& Mishkin, M. (1997). Differential effects of early hippocampal pathology on episodic and semantic memory. Science, 277, 376-380.

Williams, J. N. (2004). Implicit learning of form-meaning connections. In B. VanPatten, J. Williams, S. Rott, \& M. Overstreet (Eds.), Form meaning connections in second language acquisition (pp. 203-218). Mahwah, NJ: Erlbaum.

Williams, J. N. (2005). Learning without awareness. Studies in Second Language Acquisition, 27, 269-304. 